\title{
SHIELDING CALCULATIONS FOR A 200-MeV PROTON ACCELERATOR AND COMPARISONS WITH EXPERIMENTAL DATA $\dagger$
}

\author{
R. G. ALSMILLER, Jr., R. T. SANTORO and J. BARISH \\ Oak Ridge National Laboratory, Oak Ridge, Tennessee, 37830, USA
}

(Received January 24, 1975; in final form March 14, 1975)

\begin{abstract}
Calculated results, obtained using a combination of Monte Carlo and discrete ordinates methods, are presented and compared with experimental activation and dose-equivalent data for $200-\mathrm{MeV}$ protons incident on a thick water shield. The calculational geometry is only very approximately that used experimentally, but reasonable agreement between the calculated and experimental data has been obtained.

Calculated results of the neutron spectra produced in various angular intervals by $200-\mathrm{MeV}$ protons in thin and thick aluminum targets and the dose equivalents as a function of radius in a concrete shield when these spectra are isotropically incident at the center of the shield are also presented.
\end{abstract}

\section{INTRODUCTION}

In a series of previous papers, ${ }^{1-4}$ the results of neutron-transport calculations carried out with the method of discrete ordinates for various neutron spectra incident on a variety of thick shields have been presented. In this paper, results similar to those given previously are presented and compared with the experimental data of Distenfeld ${ }^{5}$ for $200-\mathrm{MeV}$ protons incident on a thick water shield. Results are also given for the neutron spectra averaged over various angular intervals produced when $200-\mathrm{MeV}$ protons are incident on thin and thick targets of aluminum and for the dose equivalent as a function of radius in a concrete shield when these neutron spectra are isotropically incident at the center of a spherical shield. $\ddagger$

In Section 2 the procedure used to obtain the results for comparison with the experimental data of Distenfeld ${ }^{5}$ is described and the comparisons between the calculated and experimental data are given. In Section 3 the calculational methods used to obtain the neutron spectra from $200-\mathrm{MeV}$ protons on thin and thick targets are discussed, and these spectra, together with the neutron

$\dagger$ This research was funded by the Energy and Research Development Administration under contract with the Union Carbide Corporation.

$\$$ These results were obtained to aid in the design of the shielding for the cyclotron under construction at the University of Indiana. transport results obtained using these spectra, are presented.

\section{COMPARISONS BETWEEN CALCULATED AND EXPERIMENTAL RESULTS FOR 200-MeV PROTONS INCIDENT ON A THICK WATER SHIELD}

The geometry used by Distenfeld ${ }^{5}$ to obtain his experimental data is shown schematically on the left side of Figure 1. In the experiment, $200-\mathrm{MeV}$ protons were incident on the water column, and, using activation detectors, data were taken as a function of distance measured transversely to the proton beam axis. The activation reactions considered were ${ }^{12} \mathrm{C}(\mathrm{n}, 2 \mathrm{n}){ }^{11} \mathrm{C},{ }^{27} \mathrm{Al}(\mathrm{n}, \alpha){ }^{24} \mathrm{Na}$, ${ }^{23} \mathrm{Na}(\mathrm{n}, \gamma){ }^{24} \mathrm{Na}$, and the production of ${ }^{24} \mathrm{Na}$ from neutron-induced reactions in $\mathrm{Cl}$. Experimental data on the dose equivalent above the water tank obtained with Bonner spheres have also been obtained by Distenfeld.

The geometry used to obtain the calculated results for comparison with the experimental data is shown on the right side of Figure 1. The calculational geometry is only very approximately that used experimentally in that it is assumed that

1) the neutron source which extends over the range of $200-\mathrm{MeV}$ protons in water $(\sim 25 \mathrm{~cm})$ may be considered as a point source; 


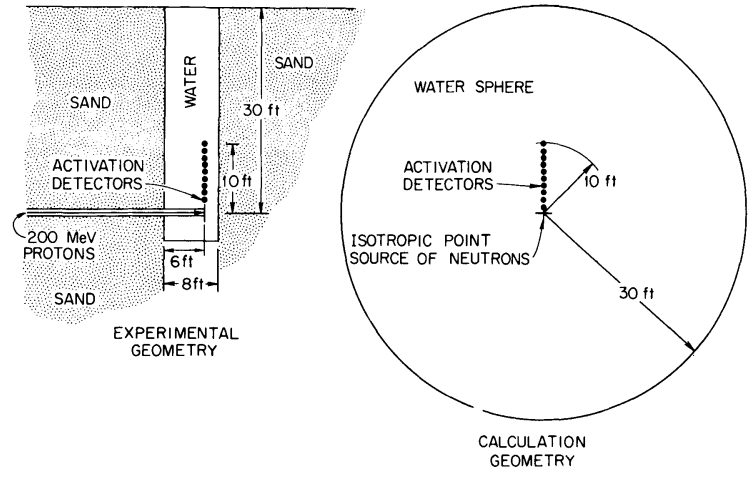

FIGURE 1 Schematic diagrams of the geometry used by Distenfeld ${ }^{5}$ in obtaining the experimental data and used in carrying out the calculations reported here.

2) the neutron source is isotropic (see discussion below); and

3) the shield is a water sphere.

The energy spectrum of the neutron source (see Figure 1) was obtained by calculating the energy distribution of neutrons produced in a specific angular interval by proton-nucleus nonelastic collisions when $200-\mathrm{MeV}$ protons are stopped in a thick water shield. The source-spectrum calculations were carried out with the Monte Carlo transport code HETC. ${ }^{6}$. This code has been described in detail elsewhere ${ }^{7}$ and therefore will not be discussed here. It is perhaps worth mentioning, however, that the source of the differential particleproduction cross-section data used in HETC at the higher energies $(>15 \mathrm{MeV})$ is the same as the source of all of the high-energy nonelastic crosssection data used in this paper-namely, the intranuclear-cascade-evaporation model of nuclear reactions as implemented by Bertini. ${ }^{8-11}$ Neutron energy spectra $(E>1 \mathrm{MeV})$ obtained with HETC for two angular intervals centered about $90^{\circ}$ with respect to the direction of the incident proton beam are shown in Figure 2. The spectra shown in the figure have been averaged over the specified angular intervals. The error bars, where shown, are statistical only and represent one standard deviation. The two energy distributions are similar, but the one corresponding to the larger angular interval contains more high-energy particles. Because of assumptions 1-3 above, it is not clear which of the two distributions should be used, and therefore calculations using both spectra will be presented and compared with the experimental data. In the calculations, the spectra shown in Figure 2 are assumed to be emitted isotropically from the point source (see Figure 1). The angular distribution of neutrons produced when $200-\mathrm{MeV}$ protons are incident on a thick water target is anisotropic and, therefore, the problem of transporting these neutrons through a spherical shield is two-dimensional; i.e., the neutron flux per unit energy in the shield is a function of both the radius into the shield and the polar angle with respect to the direction of the incident proton beam. To avoid two-dimensional calculations, the approximation of an isotropic point source is used here. The source spectrum used in the calculations is approximately correct in the vicinity of polar angles of $90^{\circ}$, and, thus, the radial dependence of the neutron flux per unit energy calculated here should be approximately the same as that which would be obtained from two-dimensional calculations in the vicinity of polar angles of $90^{\circ}$. Only incident particles above $1 \mathrm{MeV}$ were considered since the lower energy incident particles may be assumed to have no effect on the results presented.

The neutron transport calculations were carried out with the one-dimensional discrete ordinates code ANISN ${ }^{12}$ in the same manner as in Refs. 1-4. The sources of the cross-section data are the same as that given in Ref. 4. From ANISN the neutron

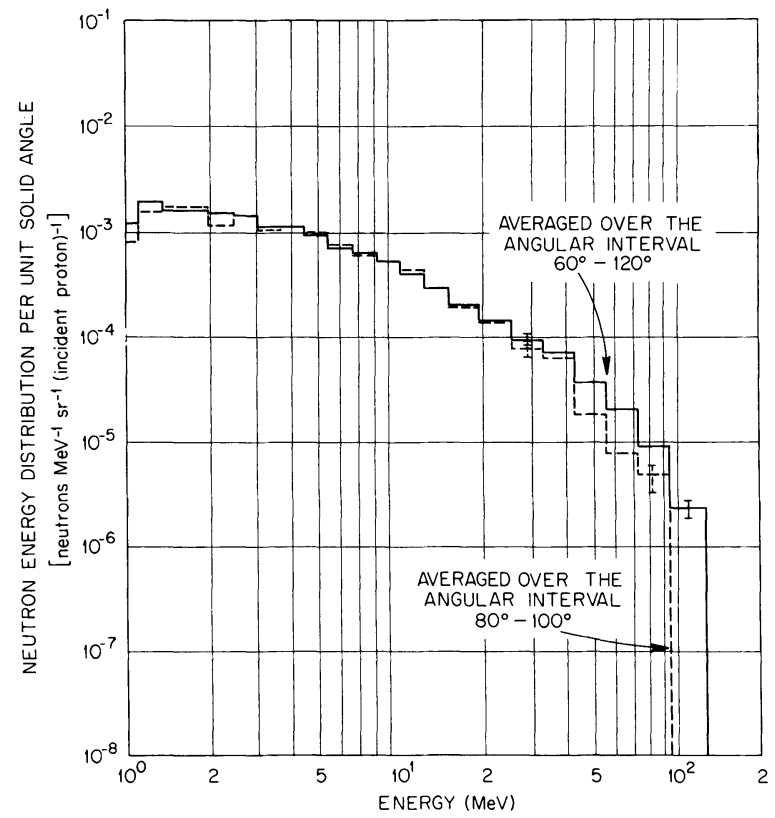

FIGURE 2 Energy distribution of neutrons, averaged over particular angular intervals, produced when $200-\mathrm{MeV}$ protons are stopped in a thick water target. (The proton beam is incident at $0^{\circ}$.) 
scalar flux per unit energy per incident proton is obtained as a function of radius in the shield, and then the neutron scalar flux per incident proton averaged over a given activation cross section is obtained from the equation

$$
\Phi_{i}(r)=\frac{\int_{E_{i}}^{E_{\max }} \Phi(E, r) \sigma_{i}(E) \mathrm{d} E}{\left[\frac{1}{E_{\max }-E_{i}}\right] \int_{E_{i}}^{E_{\max }} \sigma_{i}(E) \mathrm{d} E},
$$

where $\Phi_{i}(r)=$ the neutron scalar flux per incident proton averaged over the activation cross section of type $i$;

$\Phi(E, r)=$ the neutron scalar flux per unit energy per incident proton at radius $r$;

$\sigma_{i}(E)=$ the microscopic cross section for an activation reaction of type $i$;

$E_{i}=$ the threshold for the activation reaction of type $i$; and

$E_{\max }=$ the maximum neutron energy or the maximum energy for which $\sigma_{i}$ has an appreciable value.

The activation cross sections for the reactions ${ }^{12} \mathrm{C}(\mathrm{n}, 2 \mathrm{n}){ }^{11} \mathrm{C}$ and ${ }^{27} \mathrm{Al}(\mathrm{n}, \alpha){ }^{24} \mathrm{Na}$ were obtained as a function of energy from the work of Charalambus et $a l .{ }^{13}$ The activation cross section for the production of ${ }^{24} \mathrm{Na}$ from neutron-induced reactions in $\mathrm{Cl}$ was assumed to have a threshold of $100 \mathrm{MeV},{ }^{5}$ and to be energy-independent above this energy.

In the case of neutron capture in ${ }^{23} \mathrm{Na}$ which takes place predominantly at thermal energies, the energy-dependent cross section cannot be used to evaluate Eq. (1) because in the calculations reported here the energy dependence of the thermal-neutron flux has not been obtained. To obtain a comparison between the calculated and experimental data for the reaction ${ }^{23} \mathrm{Na}(\mathrm{n}, \gamma)^{24} \mathrm{Na}$, a very approximate procedure was used, and only a relative comparison has been obtained. It is assumed in the calculations that all of the neutron-capture reactions in ${ }^{23} \mathrm{Na}$ occur at thermal energies which are defined to be energies $\leq 0.0414 \mathrm{eV}$, and it is further assumed that $\Phi_{i}(r)$ for the reaction ${ }^{23} \mathrm{Na}(\mathrm{n}, \gamma)^{24} \mathrm{Na}$ is proportional to the neutron flux at energies $\leq 0.0414 \mathrm{eV}$; i.e.,

$$
\Phi_{i}(r)=K \Phi_{T H}(r)
$$

where $\Phi_{T H}(r)=$ the flux of neutrons with energy $\leq 0.0414 \mathrm{eV}$ at radius $r$

and $\quad K=$ a constant determined by normalizing the calculated results to the experimental data.
The dose equivalent as a function of radius into the shield was obtained using the calculated neutron scalar flux per unit energy and the flux-todose conversion factors recommended by the International Commission on Radiation Units and Measurements. $^{14}$

The calculated and experimental activation results are presented and compared in Figure 3. On the ordinate of the figure, the quantity $r^{2} \Phi_{i}$ is plotted to remove at least approximately the geometric attenuation. The experimental results shown in the figure have an absolute normalization. The calculated results, except for those given for the reaction ${ }^{23} \mathrm{Na}(\mathrm{n}, \gamma)^{24} \mathrm{Na}$, also have an absolute normalization. In the case of the ${ }^{23} \mathrm{Na}(\mathrm{n}, \gamma)^{24} \mathrm{Na}$ reaction, the calculated results have been normalized to the experimental data by multiplying the calculated thermal flux by $K=0.38$ [see Eq. (2) and the discussion pertaining to this equation].

Error bars on the experimental data in Figure 3 are not available and therefore are not shown. The calculated and experimental results are in very approximate agreement. At the larger radii, the two calculated curves bracket the experimental results. The calculated results are less valid at the smaller radii than at the larger radii because the approximation of a point source rather than an extended source is less valid at the smaller radii. It is interesting to note that at the larger radii, i.e., at radii $\gtrsim 100 \mathrm{~cm}$, the data for the reaction ${ }^{27} \mathrm{Al}(\mathrm{n}, \alpha){ }^{24} \mathrm{Na}$ agree with the calculated results when the incident spectrum is averaged over the angular interval of $80-100^{\circ}$, while the data for the reaction ${ }^{12} \mathrm{C}(\mathrm{n}, 2 \mathrm{n}){ }^{11} \mathrm{C}$ agree with the calculated results when the incident spectrum is averaged over the angular interval of $60-120^{\circ}$. Only one calculated curve is shown for ${ }^{24} \mathrm{Na}$ production from neutroninduced reactions in $\mathrm{Cl}$ because the incidentneutron spectrum averaged over the angular interval of $80-100^{\circ}$ contains no neutrons above $100 \mathrm{MeV}$ (see Figure 2), which is the threshold assumed for this type of reaction. Distenfeld ${ }^{5}$ measured a spallation reaction in $\mathrm{Cl}$ only on the sample near the source and argued that the value obtained was spurious, so this single experimental value has not been shown in the figure.

The calculated dose equivalent multiplied by the radius square is shown in Figure 4 as a function of radius in the shield. Results are given separately for the two incident spectra shown in Figure 2. Also shown in Figure 4 is the experimentally measured dose equivalent, multiplied by the radius squared, outside of the water shield. ${ }^{5}$ The experimental 


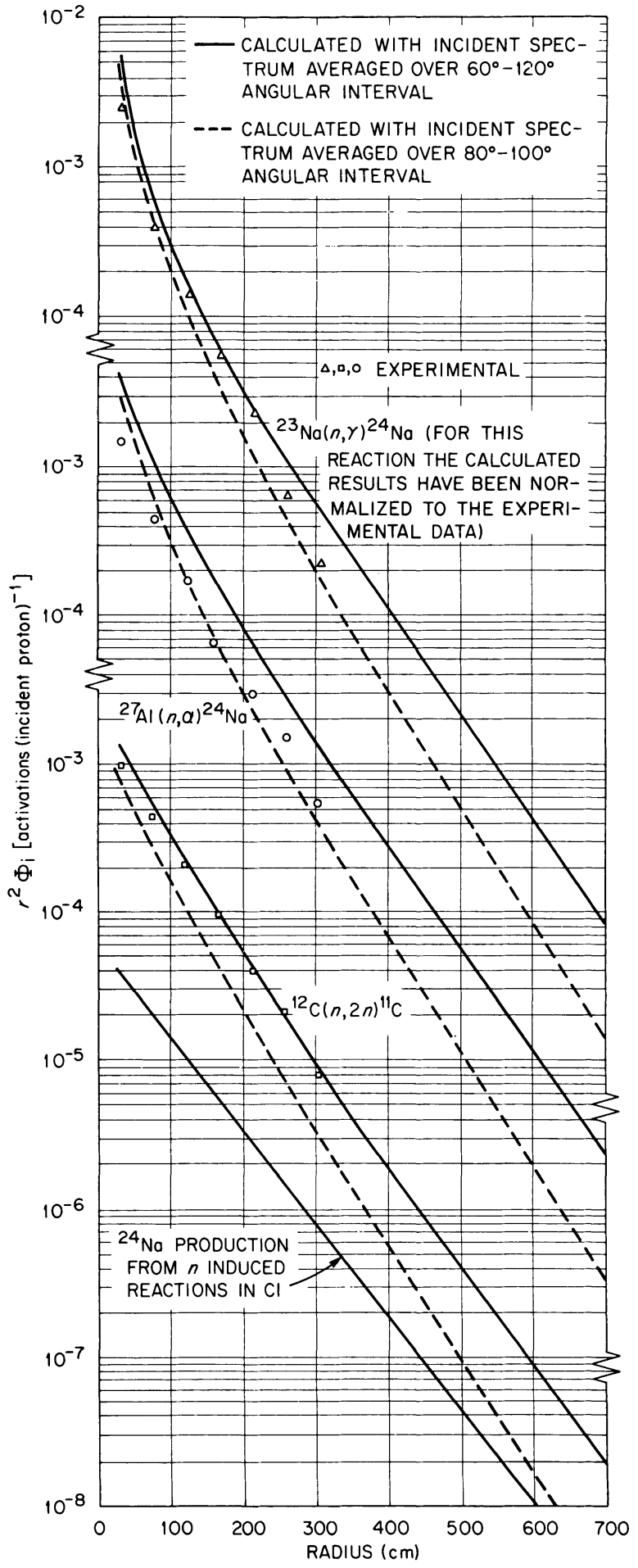

FIGURE 3 Experimental and calculated activation data multiplied by $r^{2}$ vs radius in the water shield. (The experimental data are taken from Ref. 5.)

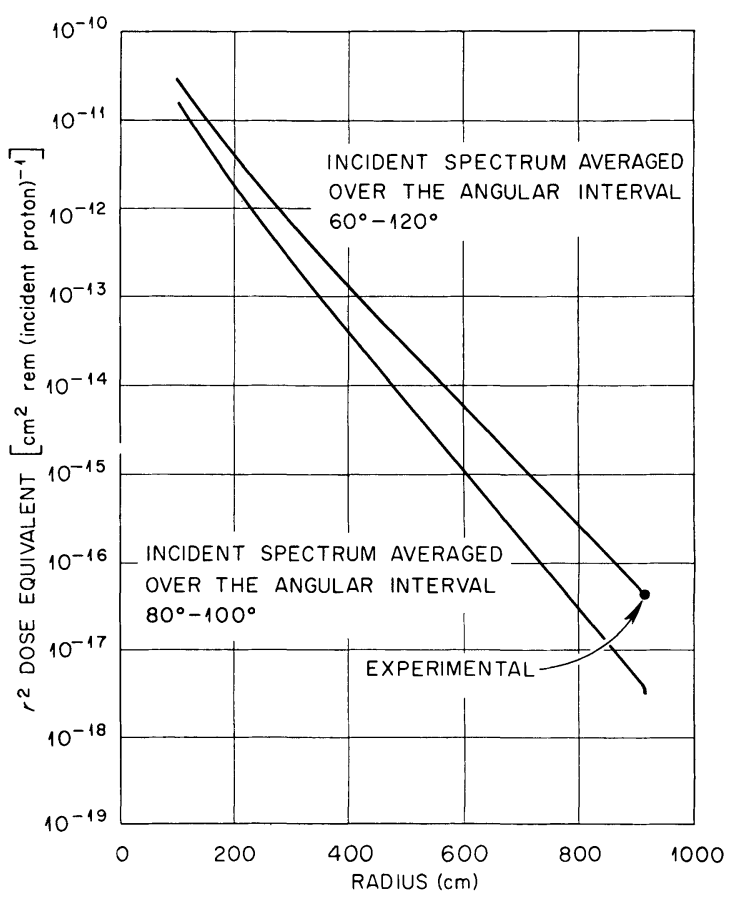

FIGURE 4 Dose equivalent vs radius in the water shield. (The incident neutron spectra used in obtaining the curves are given in Figure 2.)

result is very nearly the same as the calculated result when the incident spectrum averaged over the angular interval of $60-120^{\circ}$ is used. The rapid decrease in the calculated dose equivalents at the end of the shield is due to the absence of albedo, i.e., backscattered, neutrons at the end of the shield. It is interesting to note that the two calculated dose equivalents differ by more than an order of magnitude at the outside of the shield despite the similarity of the two incident spectra used (see Figure 2). Much of the difference in the two calculated dose equivalents is to be attributed to the neutrons above $94 \mathrm{MeV}$ which occur in only one of the incident spectra since it has been shown previously that the dose equivalent from $100-\mathrm{MeV}$ incident neutrons decreases with depth into the shield much more slowly than do the dose equivalents from incident 50 - and $75-\mathrm{MeV}$ neutrons. ${ }^{3}$

\section{CALCULATED RESULTS FOR 200-MeV PROTONS INCIDENT ON THIN AND THICK TARGETS}

A "thin" target will be taken to mean a target which is sufficiently thin that the incident protons 
may be assumed to lose no energy in passing through the target. It will further be assumed that the target dimensions are such that all neutrons produced from proton-nucleus nonelastic collisions escape from the target without undergoing nuclear interaction. Under these assumptions, the energy-angle distribution of neutrons from 200$\mathrm{MeV}$ protons on a thin target is proportional to the energy-angle distribution of neutrons from a 200-MeV proton-nucleus nonelastic collision.

A "thick" target will be taken to mean a target which is sufficiently thick that the incident protons stop in the target. The approximation is made, however, that with thick targets, as with thin targets, the neutrons produced by proton-nucleus nonelastic collisions escape from the target without undergoing nuclear interaction. The energy-angle distributions of neutrons produced by $200-\mathrm{MeV}$ protons incident on thick targets could be obtained by using HETC, as was described in Section 2, but here it was found more convenient to calculate the distributions without the use of Monte Carlo techniques. $\dagger$ The energy distribution of neutrons averaged over a specific angular interval produced by monoenergetic protons incident on a thick target was obtained from the equations

$$
\begin{aligned}
N(E)=\int_{0}^{z_{\max }} \exp ( & \left.-\int_{E^{\prime}}^{E_{0}} \frac{n \sigma\left(E^{\prime \prime}\right)}{S\left(E^{\prime \prime}\right)} \mathrm{d} E^{\prime \prime}\right) \\
& \times n \sigma\left(E^{\prime}\right) F\left[E^{\prime}\left(E_{0}, z\right), E\right] \mathrm{d} z, \\
& \int_{E^{\prime}}^{E_{0}} \frac{\mathrm{d} E^{\prime \prime}}{S\left(E^{\prime \prime}\right)}=z,
\end{aligned}
$$

where $N(E)=$ the energy distribution of neutrons averaged over a specific angular interval in neutrons $\mathrm{MeV}^{-1} \mathrm{sr}^{-1}$ (incident proton) $)^{-1}$,

$n=$ the number density of nuclei in the target in nuclei $\mathrm{cm}^{-3}$,

$\sigma(E)=$ the microscopic cross section for the nonelastic collision of a proton with energy $E$ with a target nucleus in $\mathrm{cm}^{2}$,

$F\left(E^{\prime}, E\right)=$ the energy distribution of neutrons, averaged over a specified angular interval, produced by the nonelastic

† The neutron-energy distributions used in Section 2 could not easily be calculated by the techniques used here because the differential neutron-production spectra from neutron-nucleus nonelastic collisions are available ${ }^{15,16}$ for only the angular intervals of $0-30^{\circ}, 30-60^{\circ}, 60-90^{\circ}$, and $90-180^{\circ}$, and these angular intervals are not those used in Section 2. collision of a proton with energy $E^{\prime}$ with a target nucleus in neutrons $\mathrm{MeV}^{-1} \mathrm{sr}^{-1}$ collision ${ }^{-1}\left(E<E^{\prime}<\right.$ $\left.E_{0}\right)$

$E_{0}=$ the incident proton energy in $\mathrm{MeV}$, $E^{\prime}=$ the energy in $\mathrm{MeV}$ of an incident proton at a depth $z$ in the target,

$S(E)=$ the proton stopping power in the target in $\mathrm{MeV} \mathrm{cm}{ }^{-1}$, and

$z_{\max }=$ the proton range in the target in $\mathrm{cm}$.

To obtain the thin- and thick-target results presented here, the differential neutron-production cross sections from proton-nucleus nonelastic collisions were taken from the analytic fits, given previously, ${ }^{15,16}$ to data obtained from the intranuclear-cascade-evaporation model of nuclear reactions. ${ }^{8-11}$ The proton stopping power needed to evaluate Eqs. (3) and (4) were taken from the code SPAR. ${ }^{17}$

In Figure 5 the energy distribution of neutrons $(>15 \mathrm{MeV}$ ) averaged over various angular intervals from $200-\mathrm{MeV}$ protons incident on thin and thick targets of $\mathrm{Al}$ is presented and compared. There are, of course, emitted neutrons with energies $<15 \mathrm{MeV}$, but they will not be considered here. For purposes of normalization, a thin-target thickness of 11 $\mathrm{g} \mathrm{cm}^{-2}(=0.1$ of the mean free path for $200-\mathrm{MeV}$ proton-nucleus nonelastic collisions in $\mathrm{Al}$ ) has been used in Figure 5, but the thin-target results are by assumption directly proportional to this thickness, so results for other thin-target thicknesses may easily be obtained. For both thin and thick targets, the high-energy neutrons are emitted predominantly at the smaller angles. For each of the angular intervals considered, the thin-target energy distributions decrease less rapidly with increasing energy than do the thick-target distributions. This is particularly true in the $0-30^{\circ}$ angular interval since, in this case, the thin-target distribution has a maximum in the vicinity of $175 \mathrm{MeV}$. In considering these results, it should be remembered that in a thin target the incident $200-\mathrm{MeV}$ protons are assumed to lose no energy, so all neutrons are produced by $200-\mathrm{MeV}$ proton-nucleus nonelastic collisions, while in a thick target the incident protons continually lose energy, so many of the neutrons are produced by proton-nucleus nonelastic collisions at energies considerably below $200 \mathrm{MeV}$.

Neutron-transport calculations have been carried out for each of the spectra shown in Figure 5 isotropically incident at the center of a concrete 


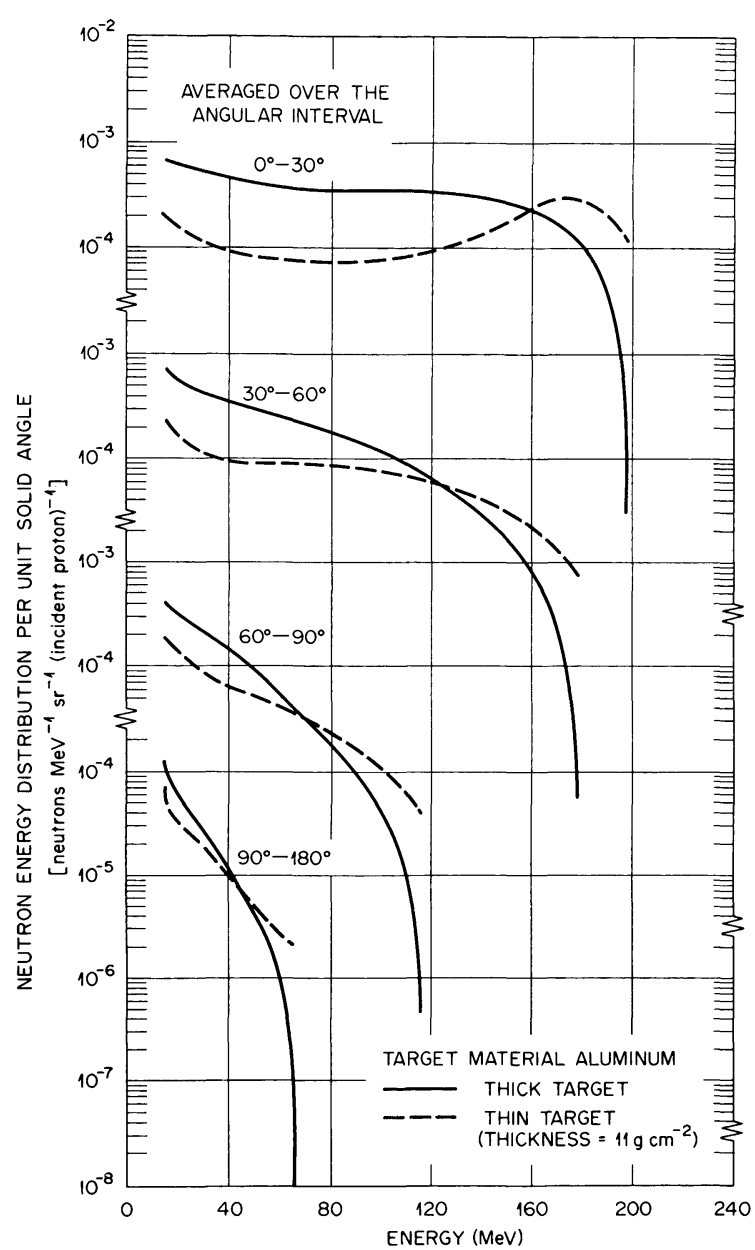

FIGURE 5 Neutron-energy distributions averaged over various angular intervals from $200-\mathrm{MeV}$ protons incident on thin and thick targets of $\mathrm{A} 1$. (The thin-target results are directly proportional to the assumed target thickness.)

shield, and the dose equivalent has been determined as a function of radius in the shield. The calculations were carried out using the discrete ordinates code ANISN in the manner described in Refs. 1-4. The composition of concrete and the source of all of the cross-section data are also the same as those used in Ref. 4. The density of concrete was taken to be $2.3 \mathrm{~g} \mathrm{~cm}^{-3}$, and the shield radius was in all cases $1500 \mathrm{~g} \mathrm{~cm}^{-2}$. The dose-equivalent calculations were carried out using flux-to-dose conversion factors ${ }^{10}$ in the same manner as in Section 2.

In Figure 6 the dose equivalent multiplied by the radius squared is shown as a function of radius into the shield for each of the spectra shown in Figure 5. In considering the results, it must be remembered that only neutrons with energies

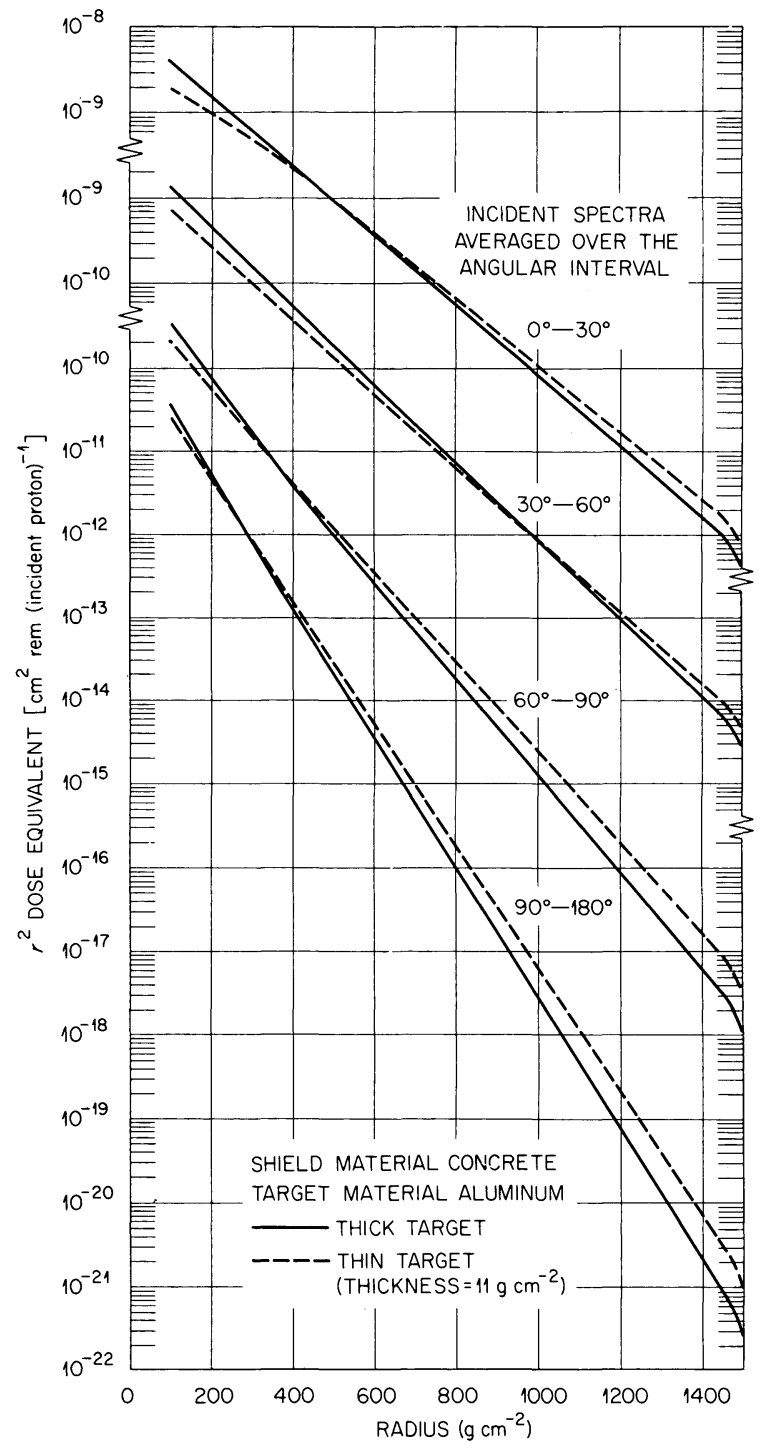

FIGURE 6 Dose equivalent multiplied by $r^{2}$ vs radius in the concrete shield. (The incident neutron spectra used in obtaining the various curves are shown in Figure 5.)

$>15 \mathrm{MeV}$ have been considered. This means that consideration must be restricted to sufficiently large distances into the shield that these lower energy incident neutrons may be assumed to make no contribution. In Figure 6 results are presented for radii $>100 \mathrm{~g} \mathrm{~cm}^{-2}$, but it is to be understood that the dose-equivalent values shown at the smaller radii might be underestimates because the lower energy incident particles have not been considered. The results for the thin target are again directly proportional to the assumed target 
thickness of $11 \mathrm{~g} \mathrm{~cm}^{-2}$. In all cases, the dose equivalent multiplied by $r^{2}$ from an incident thintarget spectrum decreases less rapidly with increasing radius into the shield than does the dose equivalent multiplied by $r^{2}$ from the corresponding incident thick-target spectrum. This is due to the fact that in a given angular interval the thin-target spectrum always contains more high-energy particles than does the thick-target spectrum. This effect of the presence of high-energy particles in the incident spectrum is also evident from the different attentuations shown in Figure 6 for the various angular intervals.

\section{REFERENCES}

1. R. G. Alsmiller, Jr., F. R. Mynatt, J. Barish, and W. W. Engle, Jr., Nucl. Sci. Eng., 36, 251 (1969).

2. R. G. Alsmiller, Jr., F. R. Mynatt, J. Barish, and W. W. Engle, Jr., Nucl. Instr. Meth., 72, 213 (1969).

3. R. W. Roussin, R. G. Alsmiller, Jr., and J. Barish, Nucl. Eng. Design, 24, 250 (1973).

4. R. G. Alsmiller, Jr., and J. Barish, Particle Accelerators, 5, 155 (1973).

5. C. H. Distenfeld, Shielding Measurements - 200 MeV Linac, Report BNL 18025, Brookhaven National Laboratory (1973).

6. K. C. Chandler and T. W. Armstrong, Operating Instructions for the High-Energy Nucleon-Meson Transport Code
HETC, Report ORNL-4744, Oak Ridge National Laboratory (1972).

7. T. W. Armstrong, R. G. Alsmiller, Jr., K. C. Chandler, and B. L. Bishop, Nucl. Sci. Eng., 49, 82 (1972).

8. H. W. Bertini, Effect of Error on Results of a Low-Energy Intranuclear Cascade Calculation, Report ORNL-3786, Oak Ridge National Laboratory (1966).

9. H. W. Bertini, Phys. Rev., 131, 1801 (1963), with erratum Phys. Rev., 138, AB2 (1965).

10. H. W. Bertini, Nucl. Phys., 87, 138 (1966).

11. H. W. Bertini, Phys. Rev., 162, 976 (1967).

12. W. W. Engle, Jr., A Users Manual for ANISN, a OneDimensional Discrete Ordinates Transport Code with Anisotropic Scattering, Report K-1693, Computing Technology Center, Union Carbide Corporation (1967).

13. St. Charalambus, J. Dutrannois, and K. Goebel, Particle Flux Measurements with Activation Detectors, Report CERN/DI/HP 90, CERN (1966).

14. International Commission on Radiation Units and Measurements, Radiation Protection Instrumentation and Its Application, ICRU Report 20 (1971).

15. R. G. Alsmiller, Jr., M. Leimdorfer, and J. Barish, Analytic Representation of Nonelastic Cross Sections and ParticleEmission Spectra from Nucleon-Nucleus Collisions in the Energy Range 25 to $400 \mathrm{MeV}$, Report ORNL-4046, Oak Ridge National Laboratory (1967).

16. R. G. Alsmiller, Jr., and J. Barish, NCDATA-Nuclear Collision Data for Nucleon-Nucleus Collisions in the Energy Range 25 to $400 \mathrm{MeV}$, Report ORNL-4220, Oak Ridge National Laboratory (1968).

17. T. W. Armstrong and K. C. Chandler, SPAR, a FORTRAN Program for Computing Stopping Powers and Ranges for Muons, Charged Pions, Protons, and Heavy Ions, Report ORNL-4869, Oak Ridge National Laboratory (1973). 